\title{
Mulheres e Homens: Diferentes Também na Percepção das Notas Musicais?
}

\author{
Jandilson Avelino da Silva ${ }^{1}$ \\ Universidade Federal da Paraíba \\ Paloma Cavalcante Bezerra \\ Universidade Federal do Piauí \\ Maria José Nunes Gadelha \\ Michael Jackson de Oliveira Andrade \\ Lisieux Marie Marinho dos Santos Andrade \\ Nelson Torro-Alves \\ Natanael Antonio dos Santos \\ Universidade Federal da Paraíba
}

\begin{abstract}
RESUMO - O objetivo deste estudo foi avaliar a percepção das notas musicais RÉ, FÁ, e LÁ em adultos jovens. Participaram do estudo 20 homens e 20 mulheres de 18 a 29 anos, saudáveis, não músicos e não usuários de fármacos ou outras substâncias tóxicas. Realizou-se um teste de discriminação de notas musicais com escolha forçada entre duas alternativas, por meio do qual os participantes escolheram um estímulo previamente apresentado. Comparou-se a frequência de acertos na discriminação das notas musicais em relação ao sexo dos participantes. Encontraram-se diferenças estatisticamente significantes na nota musical Fá, sendo que os homens apresentaram maior quantidade de acertos que as mulheres. Esses resultados encontrados sugerem diferença na percepção das notas musicais para mulheres e homens.
\end{abstract}

Palavras-chave: percepção auditiva, notas musicais, sexo

\section{Women and Men: Different Also in the Perception of Musical Notes?}

\begin{abstract}
The objective of this study was to evaluate the perception of musical notes D, F, and A in young adults. The study included 20 men and 20 women, aged 18 to 29 years, healthy, non-musicians, and non-users of drugs or other toxic substances. Participants did a two-alternative forced choice discrimination test of musical notes, in which they were asked to select the stimulus that was previously presented. The frequency of correct discrimination of the musical notes was compared between both sexes. Statistically significant differences in the musical note F were found. Men had a higher number of hits than women. These results suggest differences in the perception of musical notes by women and men.
\end{abstract}

Keywords: auditory perception, musical notes, gender

O conhecimento a respeito do processamento musical pode ser um importante referente na discussão de questões sociais, clínicas e neurobiológicas relacionadas aos aspectos auditivos (McDermott \& Oxenham, 2008; Omar, Hailstone, Warren, Crutch, \& Warren, 2010). Estudos nessa direção analisaram a habilidade de discriminação de sons, utilizando variáveis diversificadas em animais e em humanos.

Sloan, Dodd e Rennaker (2009), por exemplo, compararam a discriminação de frequências sonoras por ratos albinos em duas tarefas distintas. A primeira requisitava a detecção de uma mudança de frequência, a partir de um tom de referência permanente. A segunda requeria a detecção de uma mudança de frequência entre as reproduções de uma sequência de referência de tons repetidos. Para cada tarefa, o limiar diferencial de frequência foi medido em uma faixa de referência de 2,31 a $27,7 \mathrm{kHz}$ a $60 \mathrm{~dB}$ em nível de pressão sonora, com deslocamentos de frequência para cima e para baixo. Todos os 24 sujeitos utilizados aprenderam rapidamente a primeira tarefa, mas apenas 13 aprenderam

1 Endereço para Correspondência: Rua Walfredo Macedo Brandão, 822, Apt. 101 Jardim Cidade Universitária, João Pessoa, Paraíba. CEP 58.052-200.E-mail: jandilsonsilva@gmail.com também a segunda. O desempenho dos sujeitos em cada uma delas melhorou, em média, com o aumento da frequência de referência e, em ambas as tarefas, os limiares de mudança ascendente da frequência foram significativamente maiores do que os limiares de mudanças descendentes.

Já Walker, Schunupp, Hart-Schunupp, King e Bizley (2009) treinaram quatro furões (Mustela putorius) no método de escolha forçada entre duas alternativas, em uma tarefa de discriminação de sons que eram maiores ou menores que um som de referência, utilizando tons puros e vogais artificiais como estímulos. A tarefa dos furões era indicar se a frequência fundamental do som-alvo era maior ou menor do que a referência, ativando, respectivamente, bicos periféricos à direita ou esquerda. Cinco humanos adultos (dois do sexo masculino, com idades entre 24-40 anos) foram também testados em uma tarefa similar de discriminação de tom. Os ouvintes humanos ingênuos superaram os furões na presente tarefa, mas apontaram efeitos similares quanto ao tipo de estímulo e de referência. Esses pesquisadores sugeriram, então, que quando os animais podem ser treinados a nomear sons complexos, como mais altos ou mais baixos que determinado tom, essa tarefa pode ser muito mais difícil para os animais, em comparação aos humanos. 
Pfordresher e Brown (2009) exploraram a capacidade de utilização de tons na língua nativa para analisar as habilidades de imitação e discriminação de determinadas frequências sonoras. Eles conduziram dois estudos, sugerindo que os indivíduos cuja língua materna é uma língua tonal (na qual o tom contribui para o significado da palavra) são mais capazes de imitar e discriminar os tons musicais. Em cada estudo, o participante passava por três testes diferentes: um teste era de imitação de tons, e os outros dois, de discriminação de notas e de discriminação de intervalos, respectivamente. Para a discriminação de notas eram apresentados pares de notas musicais, sendo a primeira sempre um Dó em quinta oitava e a segunda variando entre a mesma nota ou outra diferente da primeira que se alterava em frequências de $8,15,30,61$, 122,183 , e $250 \mathrm{~Hz}$, de forma ascendente ou descendente. A discriminação de notas nos dois estudos foi melhor para os indivíduos que tinham como língua nativa uma língua tonal.

Outro estudo realizado por Brancucci, Dipinto, Mosesso e Tommasi (2009), com 52 músicos italianos voluntários (36 homens e 16 mulheres) com média de idade de 25,2 anos, analisou o fenômeno do ouvido absoluto. Esse fenômeno trata-se da capacidade de nomear o tom de um som sem o uso de qualquer referência externa. Esse estudo avaliou se quando os indivíduos erravam na identificação de um tom musical tendiam a confundir mais frequentemente o nome da nota correspondente com outro nome de nota que continha a mesma vogal (o que os experimentadores chamaram de Mesmo Erro) do que com o nome do tom que continha uma diferente vogal (chamado de Erro Diferente). Os pares de nota que podiam ser confundidos com base na vogal eram Dó-Sol, Si-Mi, Fá-Lá. O número de "Mesmos Erros" cometidos foi comparado com o número de "Erros Diferentes". Os resultados mostram que os músicos que não possuíam ouvido absoluto confundiam mais frequentemente as notas que tinham uma vogal semelhante no nome. Eles tendiam a confundir, por exemplo, um tom de $261 \mathrm{~Hz}$ (Dó) mais frequentemente com a nota Sol do que um Sol com um Lá.

Moore e Vinay (2009) testaram dois grupos de sujeitos com e sem lesões na porção basal da cóclea, região responsável por processar os sons de alta frequência. Doze indivíduos de 22 a 74 anos e diagnosticados com perda auditiva neurossensorial para frequências altas participaram do estudo. Utilizou-se o método da escolha forçada entre duas alternativas. A tarefa do sujeito era escolher a alternativa em que um som mudava ao longo de quatro apresentações dentro de um intervalo de tom. Os pesquisadores concluíram que uma região morta para altas frequências é associada com uma melhor capacidade de processar informações em baixas frequências. Esses efeitos podem refletir a plasticidade cortical induzida pela zona morta.

\section{Percepção sonora em mulheres e homens}

Determinados estudos têm focado as diferenças entre mulheres e homens. Dentre eles, alguns têm sido feitos com base nas diversidades perceptuais (Antunes \& Gouveia, 2009; Zaidan, Garcia, Tedesco, \& Baran, 2008). No entanto, poucos têm se detido na questão da percepção auditiva. Entre esses, Antunes e Gouveia (2009) verificaram o desempenho de 17 homens e 17 mulheres em uma tarefa de discriminação sonora, usando notas musicais. Cada participante escutava 20 faixas de um $C D$, cada uma delas composta por 10 sons. Cinco desses sons eram diferentes e cinco eram iguais e estavam dispostos de forma intercalada entre os sons diferentes. O primeiro dos sons era o modelo. Ainda que não tenham sido encontradas diferenças estatísticas significativas, os autores concluíram que as mulheres apresentaram melhor desempenho quando não possuíam experiência prévia na discriminação de sons. Já os homens apresentaram melhor desempenho quando eram experientes nesse aspecto.

Diferentemente, Zaidan et al. (2008), em um estudo comparativo do desempenho de 25 universitários (11 homens e 14 mulheres) nos testes de resolução temporal Random Gap Detection Test (RGDT) e Gapsin-Noise (GIN), que analisam o tempo de reação a determinadas frequências sonoras, verificaram que o sexo masculino teve um melhor desempenho quando comparado ao feminino.

Baseado nos poucos mas controversos resultados obtidos no que se refere às diferenças entre sexos, o objetivo deste estudo foi avaliar, em mulheres e homens adultos jovens, a percepção de notas musicais na quarta oitava do piano (notas RÉ, FÁ e LÁ), por meio de um teste de discriminação desses estímulos sonoros.

\section{Método}

\section{Aspectos éticos}

Para sua realização, este estudo foi aprovado por um Comitê de Ética e Pesquisa, obtendo aprovação sob o protocolo $\mathrm{n}^{\circ}$. 075/10. A participação na pesquisa aconteceu mediante a assinatura de um Termo de Consentimento Livre e Esclarecido, o qual informava os participantes sobre os objetivos do trabalho. A integração do indivíduo ao estudo ocorria de forma voluntária. Respeitava-se também a autonomia do participante, uma vez que ele podia recusar-se e/ou retirar-se da pesquisa sem prejuízo para ambas as partes, a qualquer momento. Garantiu-se ainda o anonimato do participante, assegurando a sua privacidade por meio da confidencialidade dos dados obtidos, complementando, desse modo, todos os preceitos ditados pela Resolução no ${ }^{\circ}$ 196/96 do Conselho Nacional de Saúde.

\section{Participantes}

Participaram do estudo 40 estudantes universitários de graduação e de pós-graduação (sendo 20 homens e 20 mulheres) do curso de Psicologia da Universidade Federal da Paraíba (UFPB - Campus I, João Pessoa-PB). Os voluntários encontravam-se clinicamente estáveis e sem queixas auditivas, não eram músicos e nem sabiam tocar instrumento musical algum, não estavam usando nenhum fármaco ou outra substância tóxica, e tinham idades de 18 a 29 anos de idade (Mulheres: $M=21,95 ; D P=3,14$ e Homens: $M=21,65 ; D P=2,73)$. 


\section{Instrumentos e equipamentos}

Para esta pesquisa foram utilizados: 1) um questionário composto por questões sociodemográficas (como idade, sexo, escolaridade, entre outras) e clínicas (comprometimentos físicos e psicológicos, uso de substâncias tóxicas, entre outras); 2) o PsySounds, um software gerador de notas musicais, especificamente desenvolvido para a realização deste experimento. Esse programa foi processado por um micro-computador, ao qual foram conectados uma placa de som externa da marca "Creative", modelo " $X-F i$ Surround USB 5.1 Sound Blaster" e um mouse, para indicação das respostas dos participantes aos estímulos apresentados; 3 ) dois fones de ouvidos, sendo um para o experimentador e outro para o participante. O fone do participante era da marca "Sennheiser", modelo "HD 205". Foi também utilizado um adaptador para a conexão dos fones à placa de som; e 4) uma venda de tecido que foi colocada sobre os olhos dos participantes, para evitar que estimulações visuais interferissem nas suas respostas auditivas.

\section{Estímulos}

O PsySounds foi programado em linguagem Java. Os estímulos gerados nesse software simulam as sete oitavas de um piano, dentre as quais podem ser escolhidas qualquer uma das sete notas musicais de uma escala padrão (Dó, Ré, Mi, Fá, Sol, Lá e Si). A interface de abertura do programa apresenta a possibilidade de escolha de notas musicais à direita e à esquerda. Depois de selecionadas, as notas musicais são apresentadas em pares, sendo que, em cada alternativa, apenas uma nota do lado direito da interface inicial aparece, enquanto que mais de uma nota do lado esquerdo pode surgir, sendo uma a cada vez.

Para este estudo foram escolhidas, para cada participante, em uma sessão experimental, as notas RÉ, FÁ e LÁ da quarta oitava na opção "nota teste", em comparação com as duas notas mais próximas da escala a que ela pertencia, como apresentado na Tabela 1.

\section{Procedimento}

Antes de cada sessão experimental, o participante deveria responder a um questionário, no qual eram feitas indagações sociodemográficas, como idade, sexo, lateralidade, raça, escolaridade, profissão, estado civil, renda, religião e habilidades musicais. O questionário continha ainda indagações de natureza clínica, relacionadas à saúde física e psicológica geral do participante, principalmente da saúde auditiva, e

Tabela 1.Conjunto de notas testes e distratoras.

\begin{tabular}{ll}
\hline Nota Teste & Nota Distratora \\
\hline Ré 4 & Dó 4 ou Mi 4 \\
Fá 4 & Mi 4 ou Sol 4 \\
Lá 4 & Sol 4 ou Si 4 \\
\hline
\end{tabular}

também ao uso de substâncias como tabaco, café, bebidas alcoólicas e fármacos.

As luzes do ambiente ficaram desligadas no momento do experimento. Além disso, era colocada uma venda sobre os olhos dos participantes para evitar que estimulações visuais pudessem interferir nas suas respostas auditivas. As estimativas eram realizadas com um método de escolha forçada entre duas alternativas.

Em cada teste eram apresentados, aleatoriamente, para cada participante, três grupos de 20 pares de estímulos (estímulo de teste e estímulo distrator), sendo um grupo para cada nota utilizada. A tarefa dos participantes era escolher sempre o estímulo de teste, uma nota musical padrão, denominada de "nota teste". Essa nota era apresentada para o participante, por cinco vezes consecutivas, antes que cada bloco de pares de estímulos tivesse início.

Os estímulos distratores eram sempre duas notas musicais diferentes da escolhida como teste. Elas variavam aleatoriamente entre as duas notas mais próximas da nota padrão, sendo uma localizada à direita e outra à esquerda, considerando apenas as teclas brancas de um piano. Os estímulos eram apresentados com um intervalo de um segundo entre eles. A ordem de apresentação em relação ao estímulo teste e o estímulo distrator era aleatória.

Os participantes eram instruídos a falar a palavra "primeiro", indicando que o experimentador deveria pressionar o botão esquerdo do mouse, quando julgavam que a nota musical de teste tinha sido apresentada em primeiro lugar. Quando consideravam que a nota musical de teste tinha sido apresentada em segundo lugar, deveriam dizer a palavra "segundo" e o experimentador deveria pressionar o botão direito do mouse.

Antes que cada teste tivesse início, perguntava-se ao participante se ele realmente havia entendido as instruções fornecidas. Confirmada a compreensão dos participantes sobre as instruções, começava-se o teste.

\section{Resultados}

A variável dependente no presente estudo correspondeu ao número de respostas corretas na tarefa de discriminação entre notas musicais, ou seja, ao número de vezes que o participante detectava o estímulo sonoro de teste. O número total e o número médio de respostas corretas para cada nota musical são apresentados, de acordo com o sexo dos participantes, na Tabela 2. Os valores apresentados devem ser considerados com base nas 400 respostas possíveis de cada nota musical testada ( 20 pessoas de cada sexo x 20 pares de estímulos para cada nota musical padrão).

Observa-se que o número de respostas corretas para os indivíduos do sexo masculino foi, de forma geral, superior àquele apresentado pelo sexo feminino, com exceção da nota Ré, para a qual as mulheres tiveram três respostas corretas a mais. Para os homens, a sequência do número total de respostas corretas não apresentou um padrão definido, embora tenha apresentado diferenças significativas na discriminação entre as notas musicais utilizadas. Para a nota musical Fá, os homens tiveram 17 acertos a mais em comparação às mulheres. Para a nota musical Lá, foram 10 respostas certas a mais. 
Tabela 2. Número total e média das respostas corretas obtidas no teste de discriminação de notas musicais para as mulheres e homens.

\begin{tabular}{lllllll}
\hline \multirow{2}{*}{ Sexo } & Ré & \multicolumn{3}{c}{ Fá } & \multicolumn{2}{l}{ Lá } \\
\cline { 2 - 7 } & $\begin{array}{l}\text { Respostas } \\
\text { Corretas }\end{array}$ & Média & $\begin{array}{l}\text { Respostas } \\
\text { Corretas }\end{array}$ & Média & $\begin{array}{l}\text { Respostas } \\
\text { Corretas }\end{array}$ & Média \\
\hline Mulheres & 303 & $\begin{array}{l}15,15 \\
(\mathrm{DP}=3,13)\end{array}$ & 296 & $\begin{array}{l}14,8 \\
(\mathrm{DP}=2,26)\end{array}$ & 285 & $\begin{array}{l}14,25 \\
(\mathrm{DP}=2,55)\end{array}$ \\
\hline Homens & 300 & $\begin{array}{l}15 \\
(\mathrm{DP}=2,90)\end{array}$ & 313 & $\begin{array}{l}15,65 \\
(\mathrm{DP}=2,90)\end{array}$ & 295 & $\begin{array}{l}14,75 \\
(\mathrm{DP}=2,98)\end{array}$ \\
\hline
\end{tabular}

Nota: DP = Desvio-Padrão.

Para o sexo feminino, encontraram-se diferenças significativas para discriminação entre as notas musicais, sendo que o número de respostas corretas apresentou um declínio de acordo com o aumento da frequência sonora utilizada, da nota Ré para a nota Lá. É importante saber que os valores das frequências sonoras dessas notas dão-se de forma ascendente, sendo $293.7 \mathrm{~Hz}, 349.2 \mathrm{~Hz}$ e $440.0 \mathrm{~Hz}$, respectivamente, para as notas Ré, Fá e Lá.

Um $X^{2}$ de aderência foi usado para a comparação entre o grupo de homens e de mulheres, tomados separadamente para cada uma das notas musicais utilizadas (Ré, Fá, Lá). Considerou-se como referência o número de respostas corretas na discriminação dessas notas. A escolha desse teste deu-se tanto pelos valores calculados serem números inteiros, sendo assim variáveis discretas, quanto por se tratarem de número de acertos obtidos por meio de um teste dicotômico de acertos e erros. O teste $X^{2}$ de aderência apresentou diferença significativa apenas para a nota Fá $\left(X^{2}=26,60\right.$; $g l=11 ; p=0,005)$.

\section{Discussão}

O objetivo deste estudo foi verificar a percepção das notas musicais RÉ $(293,7 \mathrm{~Hz})$, FÁ $(349,2 \mathrm{~Hz})$ e LÁ (440,0 $\mathrm{Hz}$ ) na quarta oitava do piano em mulheres e homens adultos jovens. Detectou-se que mulheres e homens, quando comparados entre si, responderam de forma diferenciada para a nota musical Fá.

Ainda que estudos relacionados ao sistema auditivo de homens e mulheres possam ser encontrados, considera-se ainda escassa a quantidade de pesquisas existentes sobre o assunto, provavelmente devido à sua complexidade estrutural e funcional. A compreensão do processamento dos estímulos sonoros, da captação externa às análises mais centrais no encéfalo, tem sido um objeto de difícil controle, o que tem gerado contradições nos resultados dos estudos encontrados, no que diz respeito ao funcionamento diferenciado da audição entre o sexo dos indivíduos, estejam eles relacionados aos mais diversos atributos (Clynes, 1982; Kraus \& Chandrasekaran, 2010; Moore, 2007; Zatorre, 2001).

Dessa forma, questões concernentes às relações entre as propriedades musicais e a percepção auditiva, tomada de forma geral, bem como as possíveis diferenças existentes na audição de mulheres e homens ainda não estão totalmente esclarecidas. Mulheres escutam padrões sonoros de forma diferente dos homens? Nesse caso, essas diferenças dão-se de formas positivas ou negativas para qual deles? É na tentativa de auxiliar na resposta para essas questões que o presente estudo está inserido, pois apesar de certos estudos utilizarem como participantes mulheres e homens, poucos têm se dedicado à comparação entre eles (Brancucci et al., 2009; Moore \& Vinay, 2009; Pfordresher \& Brown, 2009).

Em revisão da literatura realizada nas principais bases de dados nacionais e internacionais (e.g., Scielo, Pubmed, Web of Science), no ano de 2011, encontraram-se apenas dois estudos que comparavam mulheres e homens na percepção de frequências sonoras (Antunes \& Gouveia, 2009; Zaidan et al., 2008), sendo que somente um deles era relacionado a atributos musicais (Antunes \& Gouveia, 2009). Mesmo assim, nenhum desses estudos pode ser comparado diretamente ao presente manuscrito, devido a importantes diferenças metodológicas. Essa é uma lacuna instigante, pois, segundo McDermott e Oxenham (2008), o estudo dos atributos musicais pode ser relevante para a construção do conhecimento relativo aos aspectos socioclínicos e neurobiológicos ligados à audição. Além disso, para Pearson (1997), a capacidade de discriminação de frequências sonoras é especialmente importante quando se lida com sinais auditivos complexos, tais como a capacidade de entender a palavra falada ou localizar fontes sonoras. Nesse sentido, os resultados obtidos no presente estudo podem ser úteis na compreensão das funções auditivas de mulheres e homens, para, desse modo, contribuir na elaboração de estratégias avaliativas e ou interventivas que considerem aspectos particulares ao sexo das pessoas.

O estudo de Zaidan et al. (2008) encontrou, como no presente estudo, diferenças no comportamento auditivo de mulheres e homens e, do mesmo modo, os homens se sobressaíram em seu desempenho em relação às mulheres. No entanto, esses autores não compararam a discriminação das frequências sonoras musicais, mas sim, o tempo de reação dos participantes para sons específicos. Contudo, pela capacidade dos indivíduos de identificar características distintas do som dentro da faixa que corresponde ao seu espectro audível, além do tempo de reação podem ser medidos os níveis de discriminação de distintos tons, sendo reconhecido, por exemplo, uma nota musical como diferente de outra (Schiffman, 2001/2005).

Antunes e Gouveia (2009) averiguaram o desempenho em uma tarefa de percepção sonora, usando também notas musicais, do mesmo modo que o presente estudo. No entanto, esses autores utilizaram uma tarefa de identificação 
de frequências sonoras, e não de discriminação como neste estudo. No estudo de Antunes e Gouveia, os participantes tinham que identificar qual dos sons apresentados em determinadas faixas sonoras equivalia a um estímulo modelo. Eles não encontraram diferenças estatísticas significativas entre os resultados de homens e mulheres, mas concluíram que as mulheres apresentaram melhor desempenho quando não possuíam experiência prévia na discriminação de sons. Já os homens apresentaram melhor desempenho quando eram experientes. Dessa forma, os resultados de Antunes e Gouveia também não podem ser comparados diretamente com os obtidos no presente estudo, pois no presente estudo todos os participantes não possuíam qualquer experiência musical.

Deve-se levar em consideração também que os procedimentos metodológicos do presente estudo e do estudo de Antunes e Gouveia (2009) foram diferentes, tanto nos tipos de estímulos utilizados quanto na apresentação dos mesmos. Enquanto que no presente estudo utilizou-se apenas a oitava central do piano, o estudo de Antunes e Gouveia utilizou as duas oitavas centrais. Enquanto que no presente estudo um som modelo isolado deveria ser escolhido entre dois outros sons apresentados, no estudo de Antunes e Gouveia, um grupo de 10 sons era apresentado, subdividido entre cinco tons iguais e cinco tons diferentes a um determinado modelo igualmente composto por cinco tons. Dessa forma, no estudo de Antunes e Gouveia, o que deveria ser identificado era o grupo de tons, e não sons isolados.

Portanto, os resultados do presente estudo, ainda que tenham se mostrado diferentes do trabalho de Antunes e Gouveia (2009), na medida em que foram encontradas diferenças estatisticamente significativas na comparação entre o sexo dos participantes (na nota musical Fá) para a tarefa de discriminação de notas musicais, não são passíveis de uma comparação direta. Para tanto, outros estudos precisam ser realizados para analisar se essa tendência de diferenciação entre o sexo dos participantes, no que se refere à discriminação de notas musicais será mantida, o que os resultados aqui relatados já sugerem, pois mesmo em notas nas quais não se obteve diferença estatisticamente significativa, foram percebidas diferentes quantidades de acertos pelos participantes dos diferentes gêneros (ver Tabela 2).

\section{Considerações finais}

Este estudo sugere que a percepção auditiva de mulheres e homens na nota musical Fá, frequência sonora de 349,2 Hz, ocorre de forma diferenciada. Pretende-se com este estudo contribuir para as investigações em percepção auditiva, utilizando a discriminação de notas musicais para verificar o desempenho de adultos jovens em diversas condições (e.g., faixa etária, patologias físicas, deficiências, transtornos psicológicos e contato com diferentes substâncias tóxicas).

Além do uso dessas variáveis, outras sugestões para estudos posteriores são o uso da opção de tempo de reação proporcionado pelo PsySounds, importante por ser um indicador da velocidade de processamento de informações pelos indivíduos, bem como um indicador de concentração e atenção, além do uso de outras notas musicais na mesma oitava utilizada ou em diferentes oitavas, como forma de caracterizar o processamento auditivo de variadas frequências de som. O uso de diferentes oitavas possibilitará a estimulação de distintas áreas da cóclea (ligadas às baixas, médias e altas frequências sonoras).

\section{Referências}

Antunes, D. C., \& Gouveia, A., Junior (2009). Questões acerca da percepção sonora de harmônicos: a função das variáveis sexo e aprendizagem. Estudos de Psicologia, 26, 57-64.

Brancucci, A., Dipinto, R., Mosesso, I., \& Tommasi, L. (2009). Vowel identity between note labels confuses pitch identification in non-absolute pitch possessors. Plos one, 4, 6327-6333.

Clynes, M. (1982). Music, mind, and brain: The neuropsychology of music. New York: Plenum Press.

Kraus, N., \& Chandrasekaran, B. (2010). Music training for the development of auditory skills. Nature Reviews Neuroscience, 11, 599-605.

McDermott, J. H., \& Oxenham, A. J. (2008). Music perception, pitch, and the auditory system. Current Opinion in Neurobiology, 18 , 452-463.

Moore, B. C. J. (2007). Basic auditory processes. In E. B. Goldstein (Ed.), Sensation and perception (pp. 379-407). Oxford, UK: Blackwell.

Moore, B. C. J., \& Vinay, S. N. (2009). Enhanced discrimination of low-frequency sounds for subjects with high-frequency dead regions. Brain, 132, 524-536.

Omar, R., Hailstone, J. C., Warren, J. E., Crutch, S. J., \& Warren, J. D. (2010). The cognitive organization of music knowledge: A clinical analysis. Brain, 133, 1200-1213.

Pearson, P. M. (1997). The effects of ethyl alcohol on visual and auditory thresholds. Tese de doutorado não publicada, University of Western Ontario, London.

Pfordresher, P. Q. \& Brown, S. (2009). Enhanced production and perception of musical pitch in tone language speakers. Attention, Perception, \& Psychophysics, 71, 1385-1398.

Schiffman, H. R. (2005). Sensação e percepção (L. A. F. Pontes \& S. Machado, Trad.). Rio de Janeiro: LTC. (Trabalho original publicado em 2001)

Sloan, A. M., Dodd, O. T., \& Rennaker, R. L. (2009). Frequency discrimination in rats measured with tone-step stimuli and discrete pure tones. Hearing Research, 251, 60-69.

Walker, K. M. M., Schnupp, J. W. H., Hart-Schnupp, S. M. B., King, A. J., \& Bizley, J. K. (2009). Pitch discrimination by ferrets for simple and complex sounds. Journal of the Acoustical Society of America, 126, 1321-1335.

Zaidan, E., Garcia, A. P., Tedesco, M. L. F., \& Baran, J. A. (2008). Desempenho de adultos jovens normais em dois testes de resolução temporal. Pró-Fono Revista de Atualização Cientifica, 20, 19-24.

Zatorre, R. J. (2001). Neural specializations for tonal processing. Annals of the New York Academy of Sciences, 930, 193-210.

Recebido em 09.06.2012

Primeira decisão editorial em 14.06.2013

Versão final em 17.06.2013

Aceito em 25.06.2013 